\title{
AN EQUATION WITH A SINGULAR NONLINEARITY RELATED TO DIFFUSION PROBLEMS IN ONE DIMENSION*
}

\author{
BY \\ JULIO E. BOUILLET AND SÔNIA M. GOMES
}

Universidad de Buenos Aires

1. Introduction. The purpose of this work is to relate two problems of quite different origin, namely the varied initial, boundary and free-boundary problems for the similarity solution of one-dimensional diffusion and heat conduction, and the various boundary value problems for the singular "elliptic" equation in one spatial variable,

$$
-U^{\prime \prime}(x)=K(x)(U(x))^{-r}, \quad r>0, \quad U(x) \geqslant 0 .
$$

This equation can be thought of as describing steady-state temperature distribution $U(x)$ in a (one-dimensional, in our case) conducting medium in which a local voltage drop $V(x)$ is mantained; a steady current makes each point into a heat source. The electrical resistivity at $x$ is $\sigma(x)(U(x))^{r}$, where $V^{2}(x) / \sigma(x)=K(x)$, and thermal conductivity is conventionally set to be one. This is a particular case of a problem treated in [1] for the transient case, [2,3], and [4]. Equation (1) is subject to the usual boundary conditions.

Analogies between one-dimensional diffusion and boundary layer problems in fluid dynamics are well known. In Sec. 4 we show how this electrical analogy can be used in the analysis of diffusion problems.

In Sec. 2 we set up the two problems with the various boundary conditions we shall consider.

In Sec. 3 we discuss the bibliography on the two problems, and the hypotheses made on the function $K(x)$ in (1), related to the diffusivity in the diffusion context.

In Sec. 4 we give, in I, a unified uniqueness proof for problems (2), (2.1)-(2.3) below; we give, in II and III, estimates for the values $u(0)$ in (2.2) and $\eta_{1}$ in (2.3), and in IV, we include an existence proof for (1) with boundary conditions $U(0)=0, L U^{\prime}(1)=U(1)$ (cf. (4), (4.3)).

2. The equations. Consider the (generalized) diffusion equation $u_{t}=\left(k(u)\left|u_{x}\right|^{N-1} u_{x}\right)_{x}$, $N>0$. If the solution is assumed to be $u(x, t)=u\left(x / t^{1 / N+1}\right)=u(\eta)$, this equation becomes

$$
\left((N+1) k(u(\eta))\left|u^{\prime}(\eta)\right|^{N-1} u^{\prime}(\eta)\right)^{\prime}=-\eta-u^{\prime}(\eta), \quad u^{\prime}=d u / d \eta .
$$

*Received March 9, 1983. 
We shall deal with the following problems for (2)

$$
\begin{aligned}
& u(\eta) \text { satisfies }(2) \text { for } \eta \in(0,+\infty), u(0)=1, u(+\infty)=0 \\
& u(\eta) \text { satisfies }(2) \text { for } \eta \in(-\infty,+\infty), u(-\infty)=1, u(+\infty)=0
\end{aligned}
$$

$\eta_{1}>0$ and $u(\eta)$ satisfy (2) for $\eta>\eta_{1}, u\left(\eta_{1}\right)=1, u(+\infty)=0$, with the additional condition on the unknown $\eta_{1}$ and $u(\eta)$

$$
(N+1) k(1)\left|u^{\prime}\left(\eta_{1}\right)\right|^{N-1} u^{\prime}\left(\eta_{1}\right)=-L \eta_{1}, L>0
$$

Problem (2.1) corresponds to the initial-boundary value problem for $u(x, t)$ in $(0,+\infty)$, $t>0$, with data $u(0, t)=1, u(x, 0)=0$; problem $(2.2)$ is the initial value problem in $(-\infty,+\infty), t>0$, with datum $u(x, 0)=1$ if $x<0, u(x, 0)=0$ if $x>0$. Problem (2.3) is a diffusion problem in which the datum $u=1$ is attained at a moving boundary $x=\eta_{1} t^{1 / N+1}$; it was considered in [5], and [6] for $N=1$, in connection with the growth of precipitate particles by diffusion, the rate of growth being determined by the flux at the particle-matrix interface, which in turn is determined by diffusion in the region outside the particle.

The coefficient $k(s)$ is assumed positive a.e., and $(k(s))^{1 / N}$ only Lebesgue integrable in $(0,1)$ (cf. Sec. 3).

Equation (2) must be understood in a suitable weak formulation (cf. [7, 9, 10, 12, 13]). We shall proceed in a rather formal manner, which is justified if $k(u)>0$ for almost every $u$, for then $u(\eta)$ is absolutely continuous as well as $\left(k(u)\left|u^{\prime}\right|^{N-1} u^{\prime}\right)(\eta)$ (cf. [7, 9. 10]). Furthermore, $u(\eta) \geqslant 0$, non-increasing and strictly decreasing whenever positive.

Let $\eta=\eta(u)$ be the left inverse function to $u(\eta)$ where $u>0$. Then (2) gives

$$
\left((N+1) k(u)\left(-\left|\eta^{\prime}(u)\right|^{-N}\right)\right)^{\prime}=-\eta(u), \quad \eta^{\prime}=d \eta / d u .
$$

Integrating from zero to $u$ and taking into account that

$$
k(u)\left(-\left|\eta^{\prime}(u)\right|^{-N}\right)=k(u)\left|u^{\prime}(\eta)\right|^{N-1} u^{\prime}(\eta)
$$

tends to zero as $u(\eta)$ tends to zero, (cf. [7, 9]) gives

$$
-\eta^{\prime}(u)=((N+1) k(u))^{1 / N}\left(\int_{0}^{u} \eta(s) d s\right)^{-1 / N}
$$

In terms of this new unknown $\eta(u)$, problems $(2.1),(2.2),(2.3)$ become

$$
\begin{aligned}
& \eta(u) \geqslant 0 \text { near } u=0 \text { satisfies (3) in }(0,1), \eta(1)=0 \\
& \eta(u) \geqslant 0 \text { near } u=0 \text { satisfies (3) in }(0,1), \int_{0}^{1} \eta(s) d s=0 \\
& \eta(u)>0 \text { satisfies (3) in }(0,1), \int_{0}^{1} \eta(s) \mathrm{ds}=\mathrm{L} \eta(1) .
\end{aligned}
$$

The condition $\int_{0}^{1} \eta(s) d s=0$ in (3.2) follows from (3) and the fact - already mentioned — that $k(u)\left|u^{\prime}(\eta)\right|^{N-1} u^{\prime}(\eta)$ tends to zero at $\eta= \pm \infty$. The condition (3.3) comes from (3) 
and (2.3). As $L \eta(1)=\int_{0}^{1} \eta(s) d s>\eta(1)$, the necessary condition $L>1$ follows. This condition appeared as a sufficient one in [10].

Now we pass from this diffusion context to the one variable, singular elliptic one. Putting

$$
U(x)=\int_{0}^{x} \eta(s) d s, \quad U(x)>0 \quad \text { near } x=0,
$$

(3) and conditions (3.1), (3.2), and (3.3) become

$$
\begin{aligned}
-U^{\prime \prime}(x) & =((N+1) k(x))^{1 / N}(U(x))^{-1 / N}, \quad x \in(0,1) ; \\
U(0) & =U^{\prime}(1)=0 ; \\
U(0) & =U(1)=0 \\
U(0) & =0, \quad U^{\prime}(1)=L^{-1} U(1), L>1 .
\end{aligned}
$$

That is, in the context of $(1), K(x)=((N+1) k(x))^{1 / N}, r=1 / N$.

Here $U(x)$ is a continuous function in $[0,1]$, such that $U^{\prime}(x)$ is absolutely continuous locally in $(0,1)$, and (4) is satisfied almost everywhere.

The equivalence between Eq. (2) and (3) is included in [10], for $k(x)>0$ a.e. It is based on the proof that the function $u(\sigma)$, inverse to $\eta(x)$ for $\sigma$ in the image of $(0,1)$ under $\eta(x)$, is locally absolutely continuous, $\eta^{\prime}(u(\sigma)) u^{\prime}(\sigma)=1$ a.e., and

$$
\int_{0}^{u(\sigma)} \eta(s) d s=-(N+1) k(u(\sigma))\left|u^{\prime}(\sigma)\right|^{N-1} u^{\prime}(\sigma) \text { a.e. }
$$

(Problems (3) and (4) are related through a mere change of unknown function.)

To see, e.g., that (2), (2.1) is a consequence of (3), (3.1), we observe that, $u(\sigma)$ being just defined, (3.1) is equivalent to $u(0)=1$, and $\eta(0+)=+\infty$ is equivalent to $u(+\infty)=0$. If $\eta(x)$ is bounded

$$
\eta(x) \leqslant \eta(0+)=\eta_{0}<+\infty,
$$

we nevertheless have $u\left(\eta_{0}-\right)=0$, and from the equality above,

$$
\left((N+1) k(u)\left|u^{\prime}\right|^{N-1} u^{\prime}\right)\left(\eta_{0}-\right)=0
$$

as well. Therefore, setting $u(\sigma)=0$ for $\sigma \geqslant \eta_{0}$ gives the solution to (2), (2.1) in $(0,+\infty)$ as it is easily verified. The cases with boundary conditions (2.2) and (2.3) are analyzed with similar arguments. Necessary and sufficient conditions for the solution to (2) to have compact support are given in $[12,13]$ for $N=1[9,10]$.

3. Commentary. The problem (4), (4.2) is a particular situation of the one in [1], where the semilinear term $F(x, U)=K(x) U^{-r}$ (in our notation) is assumed locally Hölder continuous for $(x, U) \in(0,1) \times(0, \infty), K(x) \geqslant 0$. In [2] the mixed condition case both at $x=0$ and $x=1$ is studied, and again $K(x) U^{-r}$ is Hölder continuous at every $(x, U) \in$ $[0,1] \times(0, \infty)$. Furthermore, a condition $F(x, U) \geqslant \alpha-\beta U, \alpha, \beta>0$ is imposed. In [3], (4), (4.2) is solved under similar Hölder continuity on $F(x, U)$, but $F \geqslant 0$ and for every $x_{0} \in(0,1)$, there are $\gamma\left(x_{0}\right)>0, \rho\left(x_{0}\right)>0$ such that $K(x) \geqslant \gamma\left(x_{0}\right)$ if $\left|x-x_{0}\right|<\rho\left(x_{0}\right)$.

In [4] the hypotheses on $K(x)$ are $K(x) \in C(\bar{\Omega})(C([0,1])$ in our context), $K$ strictly positive, and the approach is that of a nonlinear eigenvalue problem. 
In fact these papers discuss existence and uniqueness of solutions for more general elliptic equations in $n$-dimensional domains, and more general semilinear terms $F(x, U)$.

The result on semilinear singular equations and diffusion equations seem obtained without any knowledge of their interrelation.

In the diffusion context (4), (4.1) was introduced, for $N=1$, in the form $-U U^{\prime \prime}=$ $2 K(x)$ in [12] for the particular case when $\int_{0}^{1}(k(s) / s) d s=+\infty$. It was also employed in [13] to treat (2), (2.2) by matching two solutions of (2.1) at $\eta=0, u^{+}(\eta)$ for $\eta>0, u^{-}(\eta)$ for $\eta<0, u^{+}(0)=u^{-}(0)=b \in(0,1)$, and obtaining $b$ by a continuity argument.

The problem (4), (4.1) was actually solved in [9] for $N>1(r<1)$, but the authors did not state this fact. In a paper soon to appear (cf. [10]), the general $N>0$ case for (3), (3.1) was considered: the function $\eta(x)=U^{\prime}(x)$ was searched for which satisfies $\eta(1)=U^{\prime}(1)$ $=\varepsilon>0$, and then $\varepsilon$ was let approach zero. Existence for (3), (3.3) was also obtained, under assumption $L>1$, but uniqueness was wanting. We shall prove this in Sec. 4 .

We observe in passing that a free boundary problem, (2), (2.3), became a fixed boundary, mixed problem for the inverse function integrated, a device employed in [11].

The hypotheses on $K(x)$ are less restrictive in the diffusion context (and, of course, one spatial dimension). The assumptions are $K(x)=((N+1) k(x))^{1 / N}$ integrable in $(0,1)$ for the papers [12, 13, 7] (case $N=1$ ) [9, 10]. In [12, 13], moreover, $K(x)$ is strictly positive if $x>0$. If (2) is obtained from a (generalized) heat conduction equation

$$
(E(w))_{t}=\left(k_{1}(w)\left|w_{x}\right|^{N-1} w_{x}\right)_{x}
$$

via the enthalpy variable $u=E(w)$, then

$$
k(u)=k_{1}(H(u))\left(H^{\prime}(u)^{N}\right),
$$

with $H(u)$ the left inverse function to $E$. Thus if $E$ has a jump at $w_{0}, E\left(w_{0}-\right)=a$, $E\left(w_{0}+\right)=b$, then $H^{\prime}(u)=0$ in $(a, b)$ and so $k(u)=0$ in $(a, b)$. Therefore intervals where $K=0$ can be related to changes of phase of a medium undergoing a heat conduction process, as in the case of the classical Stefan problem $(N=1)$ with one or many phases, which is thus included in the presentation (2), (2.1) if $k(u) \geqslant 0$. However, the similarity solution $u(\eta)$ cannot be expected continuous now, and the equivalence between (2) and (3) seems more difficult to establish.

Thus it is sensible to assume $K(x) \geqslant 0$ (intervals where $K=0$ included). This was done in the communications [8] and [14], for (3), (3.1) and $N=1$, and is the subject-matter of a paper in progress. The solutions obtained are such that $\eta(x)=U^{\prime}(x)$ is absolutely continuous locally in $(0,1)$, with $U^{\prime}(x)$ constant on intervals where $K(x)=0$.

It should be observed that for (4), (4.1), (4.2), $N>0$, this hypothesis $K(x) \geqslant 0$ was actually employed in [1], together with the hypotheses already mentioned. In [15], under the hypotheses $K(x) \geqslant 0$ (intervals where $K=0$ included), and $(x(1-x))^{-1 / N} K(x) \in$ $L^{1}(0,1), N>0$, the existence of $U(x) \in C^{1}([0,1])$ solution of (4), (4.2) is obtained in the form $U(x)=x(1-x) \Psi(x)$. The new unknown $\Psi(x)$, bounded away from zero, is searched for in $C([0,1])$. With this device the non-linear singularity of the equation is avoided and information on the behaviour of the solution near the boundary is obtained.

Problem (4), (4.3) can be analyzed with the same approach. This shall be done in Sec. 4. Some results on boundary behaviour have already been obtained in [4] and [12]. 
4. Applications. We show in this Section how the approach (4), ., (4.3) can be used in the proof of certain results pertaining to the diffusion context.

I. Theorem. If $0 \leqslant K(x)=((N+1) k(x))^{1 / N} \in L^{1}(0,1)$, then problems (4), (4.1), (4.2), (4.3) have at most one solution.

Proof. We point out first that existence and uniqueness for (4), (4.1) are consequences of the same for (4), (4.2) considered in $(0,2)$ with $k(x)$ replaced with $\tilde{k}(x)=k(x)$ if $0 \leqslant x \leqslant 1, \dot{k}(x)=k(2-\mathrm{x})$ if $1 \leqslant x \leqslant 2$. Then $U(x)$ is symmetric by uniqueness - proved below - and therefore $U^{\prime}(1)=0$. In the diffusion context (4), (4.1) was treated first, as remarked in Sec. 3 (cf. also [13]).

Let $U, V$ be solutions with locally absolutely continuous derivatives of (4), and $U(s)=V(a), U(b)=V(b), 0 \leqslant a<b \leqslant 1$; we shall prove that $U=V$ in $[a, b]$. Without loss of generality we may assume $U \leqslant V$ in $[a, b]$, hence

$$
-(U-V)^{\prime \prime}=K(x)\left((U(x))^{-1 / N}-(V(x))^{-1 / N}\right) \geqslant 0 \quad \text { a.e. in }(a, b)
$$

But $U-V \leqslant 0$ in $[a, b]$ with equality at the end-points and $(U-V)^{\prime \prime} \leqslant 0$ a. e. imply $U=V$ in $[a, b]$.

Therefore uniqueness for (4), (4.2) is proved. For (4), (4.3) it remains to exclude the case $U<V$ in $(a, 1], 0 \leqslant a, U(a)=V(a)$. Multiplying (5) times $x$, integrating by parts in $(a, 1)$, and using (4.3) gives

$$
\begin{aligned}
0 & \leqslant \int_{a}^{1} x K(x)\left((U(x))^{-1 / N}-(V(x))^{-1 / N}\right) d x \\
& =a\left(U^{\prime}(a)-V^{\prime}(a)\right)+\left(L^{-1}-1\right)(V(1)-U(1)) .
\end{aligned}
$$

The inequality $a\left(U^{\prime}(a)-V^{\prime}(a)\right) \leqslant 0$ is easily established for $a \geqslant 0$, and $\left(L^{-1}-1\right)(V(1)$ $-U(1))<0$ due to $L>1$. We have thus obtained a contradiction. Corollary. If $0 \leqslant$ $((N+1) k(x))^{1 / N} \in L^{1}(0,1)$, then problems (2), (2.1), (2.2), (2.3) have at most one solution, if $k(x)>0$ a.e.

We present some estimates for parameters relevant to problems (4), (4.2) and (4.3).

II. Consider (4), (4.2). There is a $b$ where $U^{\prime}(b)=0$, which is unique if $k(x)>0$ a.e. Clearly $b=u(0)$ in (2), (2.2), and is the asymptotic value of $u(x, t)=u\left(x / t^{1 / N+1}\right)$ as $t \rightarrow+\infty$, so bounds for $b$ are important. We prove first the following inequalities. We put $K(x)=((N+1) k(x))^{1 / N}$ as before, and assume all integrands in $L^{1}(0,1)$ (for instance, take $N \geqslant 1$ ) (cf. 13] for $N=1$ ).

$$
\begin{gathered}
f_{1}(b):=\int_{0}^{b} x K(x) d x \leqslant(U(b))^{1+1 / N} \\
\leqslant b^{1 / N} \int_{0}^{b} x^{1-1 / N} K(x) d x=: f_{2}(b) ; \\
g_{1}(b):=\int_{b}^{1}(1-x) K(x) d x \leqslant(U(b))^{1+1 / N} \\
\leqslant(1-b)^{1 / N} \int_{b}^{1}(1-x)^{1-1 / N} K(x) d x=: g_{2}(b) .
\end{gathered}
$$


The inequality (6.1) follows from

$$
\begin{aligned}
U(b) & =\int_{0}^{h} U^{\prime}(x) d x=\int_{0}^{b} \int_{x}^{h} K(s)(U(s))^{-1 / N} d s d x \\
& =\int_{0}^{b} x K(x)(U(x))^{-1 / N} d x,
\end{aligned}
$$

after observing that $U(b) \geqslant U(x), U(b) x / b \leqslant U(x)$ in $(0, b)$ due to the fact that $U^{\prime \prime}(x) \leqslant 0$. Replacing in (7) gives (6.1). To obtain (6.2) one applies the same considerations to

$$
U(b)=-\int_{b}^{1} U^{\prime}(x) d x, \quad U(b) \geqslant U(x), \quad U(b)(1-x) /(1-b) \leqslant U(x) \quad \text { in }(b, 1) .
$$

Observe now that the functions of $b, f_{1}$ and $f_{2}$ are monotone increasing, $f_{1}(0)=f_{2}(0)=0$, and $g_{1}, g_{2}$ are monotone decreasing, $g_{1}(1)=g_{2}(1)=0$. Furthermore, $f_{1}(x) \leqslant f_{2}(x), g_{1}(x)$ $\leqslant g_{2}(x)$ in $(0,1)$, and from $(6.1),(6.2)$ we also have $f_{1}(b) \leqslant(U(b))^{1+1 / N} \leqslant g_{2}(b), g_{1}(b) \leqslant$ $(U(b))^{1+1 / N} \leqslant f_{2}(b)$. From these observations it follows that if $a$ is the solution of the equation $f_{2}(x)=g_{1}(x)$, and $c$ is the solution of the equation $f_{1}(x)=g_{2}(x), a, c \in(0,1)$, then $a \leqslant b \leqslant c$ as desired.

III. Consider (4), (4.3) The value $U^{\prime}(1)=\eta(1)=\eta_{1}$ gives the speed of advance of the free boundary $x=\eta_{1} t^{1 / N+1}$ in (2), (2.3), i.e., the rate of growth of the particle. To obtain bounds for $U^{\prime}(1)$ we proceed as in II., observing that from (4.3)

$$
\begin{aligned}
(L-1) U^{\prime}(1) & =U(1)-U^{\prime}(1)=\int_{0}^{1}\left(U^{\prime}(x)-U^{\prime}(1)\right) d x \\
= & \int_{0}^{1} \int_{x}^{1} K(s)(U(s))^{-1 / N} d s d x=\int_{0}^{1} x K(x)(U(x))^{-1 / N} d x
\end{aligned}
$$

But $U^{\prime \prime}(x) \leqslant 0$, and so $U^{\prime}(x)$ decreases to $U^{\prime}(1) \geqslant 0$. Therefore

$$
U(1) x \leqslant U(x) \leqslant U(1) .
$$

Replacing these two bounds in place of $U(x)$ in (8) gives

$$
\left(L^{1 / N}(L-1)\right)^{-1} \int_{0}^{1} x K(x) d x \leqslant\left(U^{\prime}(1)\right)^{1+1 / N} \leqslant\left(L^{1 / N}(L-1)\right)^{-1} \int_{0}^{1} x^{1-1 / N} K(x) d x .
$$

IV. We shall give here an existence proof for (4), (4.3).

THEOREM. If $x^{-1 / N} K(x) \in L^{\prime}(0,1), K(x) \geqslant 0$, then (4), (4.3) has a unique solution $U(x)$, and $U(x) \in C^{1}([0,1])$.

Proof. The uniqueness has already been proved in I theorem. For the existence we first observe that if $f \in L^{1}(0,1)$ and $v(x)=\int_{0}^{1} g(x, s) f(s) d s$, where

$$
g(x, s)= \begin{cases}x(1+s /(L-1)), & 0 \leqslant x<s \\ s(1+x /(L-1)), & s \leqslant x<1,\end{cases}
$$

then $v \in C^{1}([0,1]), v^{\prime}(x)=\int_{0}^{1} D_{x} g(x, s) f(s) d s, v(x)$ verifies (4.3) and $-v^{\prime \prime}(x)=f(x)$ a.e. Hence, if a function $U(x)$ is such that $U(x) \geqslant C x, C>0$, and $U(x)=$ $\int_{0}^{1} g(x, s) K(s)(U(s))^{-1 / N} d s$, then $K(s)(U(s))^{-1 / N} \in L^{1}(0,1)$ and, from the observation above, the function $U(x) \in C^{1}([0,1])$ is a solution to (4), (4.3). 
Therefore to prove the theorem it is enough to determine a function $\Psi(x)$, strictly positive, that verifies

$$
\Psi(x)=x^{-1} \int_{0}^{1} g(x, s) K(s) s^{-1 / N}(\Psi(s))^{-1 / N} d s .
$$

Consequently, we must solve the fixed point problem $\Psi=T \circ F(\Psi)$, where $F(\Psi):=\Psi^{-1 / N}$ and $T w(x):=\int_{0}^{1} N(x, s) w(s) d s$, with

$$
N(x, s)=\left\{\begin{array}{l}
x^{-1} g(x, s) s^{-1 / N} K(s), \quad 0<x \leqslant 1, \\
K(s)(1+s /(L-1)) s^{-1 / N}, \quad x=0 .
\end{array}\right.
$$

To solve this problem we proceed as in the proof of theorem (1.5) of [15]. First we prove the following statements

There is an $R \geqslant 1$ such that $1 / R \leqslant \int_{0}^{1} N(x, s) d s \leqslant R$,

$T: C([0,1]) \rightarrow C([0,1])$ is a completely continuous operator.

It is clear that for all $x_{0} \in[0,1]$,

(a) $\lim _{x \rightarrow x_{0}} N(x, s)=N\left(x_{0}, s\right)$, and

(b) $\lim _{x \rightarrow x_{0}} \int_{0}^{1} N(x, s) d s=\int_{0}^{1} N\left(x_{0}, s\right) d s$.

That is, $m(x):=\int_{0}^{1} N(x, s) d s$ is a positive continuous function in $[0,1]$. Hence $m(x)$ is bounded and bounded away from zero, and (9.1) is proved. From (a) and (b) we have also

$$
\lim _{x \rightarrow x_{0}} \int_{0}^{1}\left|N(x, s)-N\left(x_{0}, s\right)\right| d s=0 \quad \text { for all } x_{0} \in[0,1]
$$

Therefore $T$ is a completely continuous operator.

Now, for $0<\varepsilon \leqslant 1$ we define

$$
f_{\varepsilon}(x)=\left\{\begin{array}{l}
\varepsilon^{-1 / N}, \quad 0 \leqslant x \leqslant \varepsilon \\
x^{-1 / N}, \quad x \geqslant \varepsilon
\end{array}\right.
$$

and $F_{\varepsilon}(w)(x)=f_{\varepsilon}(w(x))$. Putting $\quad w_{1}(x)=R^{-1-1 / N} \varepsilon^{1 / N^{2}}, \quad w_{2}(x)=R \varepsilon^{-1 / N}$, and $\left[w_{1}, w_{2}\right]:=\left\{w \in C([0,1]): w_{1}(x) \leqslant w(x) \leqslant w_{2}(x)\right\}$, and as a consequence of $(9.1)$ and (9.2) we have

$$
T \circ F:\left[w_{1}, w_{2}\right] \rightarrow\left[w_{1}, w_{2}\right] \text { is continuous and compact. }
$$

As in the proof of the Lemma (1.4) of [15], the existence of a unique $\Psi_{\varepsilon} \in\left[w_{1}, w_{2}\right]$ such that $T \circ F_{\varepsilon}\left(\Psi_{\varepsilon}\right)=\Psi_{\varepsilon}$ is a consequence of the maximum principle and of Schauder's fixed point theorem. Furthermore $\Psi_{\varepsilon} \geqslant \Psi_{\varepsilon^{\prime}}$ if $0<\varepsilon<\varepsilon^{\prime}$. Therefore if $\varepsilon=R^{-1-1 / N}, \Psi_{\varepsilon} \geqslant \Psi_{1} \geqslant \varepsilon$. This implies that $T \circ F\left(\Psi_{\varepsilon}\right)=T \circ F_{\varepsilon}\left(\Psi_{\varepsilon}\right)=\Psi_{\varepsilon}$. Hence $U(x)=x \Psi_{\varepsilon}(x)$ is the desired solution.

\section{REFERENCES}

[1] W. Fulks and J. S. Maybee, A singular nonlinear equation, Osaka Math. J. 12 (1960), 1-19

[2] C. A. Stuart, Existence theorems for a class of nonlinear integral equations, Math. Z. 137 (1974), 49-66

[3] C. A. Stuart, Existence and approximation of solutions of nonlinear elliptic equations, Math. Z. 147 (1976), $53-63$ 
[4] M. G. Crandall, P. H. Rabinowitz, and L. Tartar, On a Dirichlet problem with a singular nonlinearity, TSR 1680, University of Wisconsin, 1976

[5] C. Atkinson and C. W. Jones, Similarity solutions in some nonlinear diffusion problems and in boundary-layer flow of a pseudo plastic fluid, Q. J. Mech. Appl. Math. XXVII (1974), 193-211

[6] C. Atkinson, Some asymptotic results for the effect of composition-dependent diffusion on the growth rate of precipitate particles, Q. J. Mech. Appl. Math. XXVII (1974), 299-316

[7] J. E. Bouillet, D. A. Saravia and L. T. Villa, Similarity solutions of the equation of one-dimensional heat conduction, J. Diff. Equations 35 (1980), 55-65

[8] D. A. Saravia and J. E. Bouillet, Aproximación lineal por trozos de las soluciones de similaridad de la ecuación de la difusion, con coeficiente dependiente de la concentracion, Communication to the 1980 Meeting of the U.M.A., Rev. Un. Mat. Argentina 30, (1981) 55-56

[9] C. Atkinson and J. E. Bouillet, Some qualitative properties of solutions of a generalised diffusion equation, Math. Proc. Camb. Phil. Soc. 86 (1979), 495-510

[10] J. E. Bouillet and C. Atkinson, A generalized diffusion equation: Radial symmetries and comparison theorems, J. Math. Anal. Appl. (to appear)

[11] J. E. Bouillet, Heat-flux comparison based on properties of the medium, Adv. Appl. Math. (1981), 76-90

[12] F. V. Atkinson, and L. A. Peletier, Similarity solutions of the nonlinear diffusion equation, Arch. Rat. Mech. Anal. 54 (1974), 374-392

[13] C. J. van Duyn and L. A. Peletier, A class of similarity solutions of the nonlinear diffusion equation, Nonl. Anal., Theory, Methods Appl. 1 (1977), 223-233

[14] J. E. Bouillet, J. P. Milaszewicz and D. A. Saravia, On approximate similarity solutions of one dimensional diffusion equations, Communication to the SIAM 30th. Anniversary Meeting, July 1982

[15] S. M. Gomes, Existência e comportamento na fronteira de solucão de problema de Dirichelt não linear com singularidade na fronteira. Dissertation, Instituto de Matemática Pura e Aplicada $-\mathrm{CNPq}-$, Rio de Janeiro, December 1982 